\title{
Predicting long-term sickness absence among employees with frequent sickness absence
}

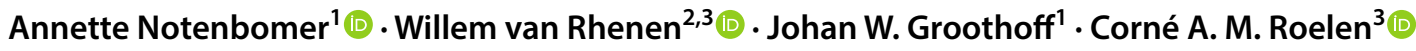

Received: 14 May 2018 / Accepted: 4 November 2018 / Published online: 24 November 2018

(c) The Author(s) 2018

\begin{abstract}
Purpose Frequent absentees are at risk of long-term sickness absence (SA). The aim of the study is to develop prediction models for long-term SA among frequent absentees.

Methods Data were obtained from 53,833 workers who participated in occupational health surveys in the period 2010-2013; 4204 of them were frequent absentees (i.e., employees with $\geq 3$ SA spells in the year prior to the survey). The survey data of the frequent absentees were used to develop two prediction models: model 1 including job demands and job resources and model 2 including burnout and work engagement. Discrimination between frequent absentees with and without long-term SA during follow-up was assessed with the area under the receiver operating characteristic curve (AUC); (AUC) $\geq 0.75$ was considered useful for practice.

Results A total of 3563 employees had complete data for analyses and 685 (19\%) of them had long-term SA during 1-year follow-up. The final model 1 included age, gender, education, marital status, prior long-term SA, work pace, role clarity and learning opportunities. Discrimination between frequent absentees with and without long-term SA was significant (AUC 0.623; 95\% CI 0.601-0.646), but not useful for practice. Model 2 showed comparable discrimination (AUC 0.624; 95\% CI 0.596-0.651) with age, gender, education, marital status, prior long-term SA, burnout and work engagement as predictor variables. Differentiating by gender or sickness absence cause did not result in better discrimination.

Conclusions Both prediction models discriminated significantly between frequent absentees with and without long-term SA during 1-year follow-up, but have to be further developed for use in healthcare practice.
\end{abstract}

Keywords Absenteeism $\cdot$ Sick leave $\cdot$ Prediction model $\cdot$ ROC analysis $\cdot$ Occupational health $\cdot$ Health surveillance

\section{Introduction}

Frequent sickness absence (SA), that is three or more SA spells per year, is usually not considered an important problem because most of the time, frequent absentees are not long off work. However, previous research has shown

Annette Notenbomer

annette.notenbomer@gmail.com

1 Division Community and Occupational Medicine, Department of Health Sciences, University Medical Center Groningen, Rijksuniversiteit Groningen, Gezondheidswetenschappen, sectie Sociale Geneeskunde, Antonius Deusinglaan 1, 9713 AV Groningen, The Netherlands

2 Center for Leadership and Management Development, Business University Nyenrode, Breukelen, The Netherlands

3 Arbo Unie, Utrecht, The Netherlands that frequent SA is risk factor of long-term SA. Koopmans et al. (2008a) reported that $19 \%$ of the frequent absentees had long-term SA (i.e., 42 consecutive days or longer) in the first year of a 4-year follow-up study. During the whole 4-year follow-up period, $50 \%$ of the frequent absentees had long-term SA.

Few studies have investigated the potential risk factors of long-term SA among frequent absentees; we only found studies with employees with frequent SA as subgroups. Women with frequent SA were reported to have a higher risk of long-term SA than men with frequent SA (Koopmans et al. 2008a, b). Furthermore, frequent absentees with prior long-term SA were shown to have a higher risk of long-term SA during follow-up (Koopmans et al. 2008a, b; Stapelfeldt et al. 2014). Stapelfeldt et al. (2014) also investigated work characteristics as risk factors of long-term SA. Work pace, emotional demands, demands for hiding emotions, physical workload, influence, meaning of work, commitment to 
the workplace, role conflict and quality of leadership were dichotomized into favorable versus unfavorable and then summed into a score of unfavorable work factors. A higher score of unfavorable work factors was associated with an increased risk of long-term SA, but the authors did not specify the results for frequent absentees. Furthermore, the associations between individual work characteristics and long-term SA were not investigated.

Psychosocial work characteristics are known predictors of long-term SA (Stapelfeldt et al. 2014; Strømholm et al. 2015; Clausen et al. 2014; Borritz et al. 2010). Various theoretical models have been developed to explain the relationship between psychosocial work characteristics and SA. The Job Demands-Resources (JD-R) model is one of those theoretical models that allows a broad range of job demands (i.e., aspects of the job that require physical and/or psychological effort) and job resources (i.e., aspects of the job that are supportive for achieving goals and/or stimulate personal development) (Bakker and Demerouti 2007). The JD-R model posits a health impairment process, in which sustained high job demands lead to burnout and long-term SA (Clausen et al. 2012, 2014; Bakker et al. 2003; Slany et al. 2014). Sustained low job resources are associated with poor work engagement and both frequent and long-term SA (Clausen et al. 2014; Borritz et al. 2010; Slany et al. 2014; Schaufeli and Bakker 2004, 2009; Roelen et al. 2015; Rongen et al. 2015). There is some evidence that burnout lies on the pathway between job demands and long-term SA. Schaufeli et al. (2009) reported that an increase in job demands and a decrease in job resources predicted burnout, and that burnout predicted longer SA duration. Eriksson et al. $(2008,2011)$ described a burnout stair case, with job demands and job resources at the lower staircases, followed by burnout and SA at the highest staircase. We are not aware of earlier predictor model studies to predict long-term SA in a population of employees with frequent SA.

The aim of the present study was to develop a prediction model for long-term SA among frequent absentees based on the predictor variables retrieved from the literature and the theoretical framework of the JD-R model, to enable timely prevention of long-term SA. If burnout and work engagement lie on the pathway between job demands and job resources on the one hand and long-term SA on the other, it would not be appropriate to include burnout and work engagement in a prediction model together with job demands and job resources (Fig. 1).

Therefore, we developed two prediction models: model 1 , including job demands and job resources, not burnout nor work engagement and model 2 including burnout and work engagement, without job demands and job resources. We compared the models for their ability to predict longterm SA among frequent absentees. Job demands and job resources are likely to differ across work settings and it may be unfeasible to capture all potentially important job demands and job resources in the prediction model. Based on the JD-R model's health impairment and motivational process, unmeasured job demands and job resources will also increase or reduce burnout and work engagement levels. Therefore, we hypothesized that a prediction model including burnout and work engagement instead of job demands and resources would better predict long-term SA among frequent absentees than a prediction model with job demands and job resources. For both models, we tested model performance of predicting long-term SA differentiating by gender and sickness absence cause.
Fig. 1 The job demandsresources (JD-R) model

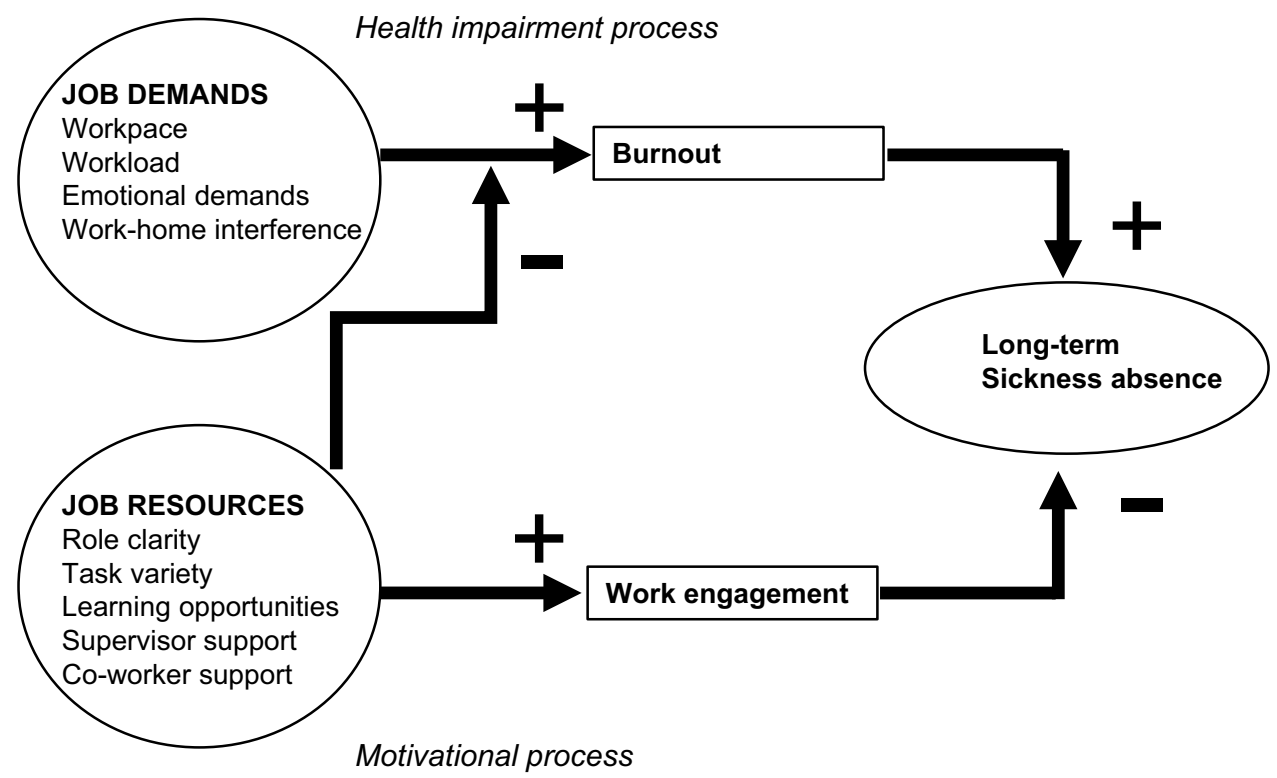




\section{Methods}

\section{Study setting and design}

Surveillance of work and health is an important OHS task in The Netherlands. According to Dutch law, employers have to enable their personnel to participate in occupational health surveys every 4 years.

In the period between 2010 and 2013, 53,833 employees, working in companies across a wide range of economic sectors contracted by a large Dutch occupational health service, participated in occupational health surveys and completed questionnaires measuring health-related and work-related variables. The response rate varied across surveys between $40-60 \%$. The survey participants (79\% men) had a mean age of 45.1 [standard deviation (SD) 10.4] years and were working $38.1(\mathrm{SD}=7.1) \mathrm{h}$ per week for on average $15.7(\mathrm{SD}=12.0)$ years in agriculture $(3 \%)$, industry $(71 \%)$, commercial services $(14 \%)$, and public services $(12 \%)$.

A total of 4204 (8\%) employees participating in the occupational health surveys were eligible for the present study because they were frequent absentees in the sense that they had three or more SA spells in the year prior to the survey. The study was set up as an explorative cohort study based on a convenience sample in which baseline variables retrieved from the occupational health survey questionnaires were analysed against SA data recorded in the year following the survey. A total of 641 frequent absentees with missing responses on baseline predictor variables were excluded from the analyses. Consequently, complete cases analysis included the data of 3563 frequent absentees. The Medical Ethics Committee of the University Medical Center Groningen granted ethical clearance for this study (M12.116654).

\section{Outcome variable long-term SA}

SA refers to a paid leave from work due to any (i.e., workrelated as well as non-work-related) illness or injury. SA was recorded in an occupational health register from the day of reporting sick to the day of returning to work. In The Netherlands, SA has to be certified by an occupational physician (OP) if it lasts 42 days or longer. Therefore, we defined SA lasting $\geq 42$ consecutive days as long-term SA, irrespective of cause. Long-term SA was obtained from the occupational health register in the year following the occupational health survey. Causes of long-term SA at follow-up were based on diagnoses of occupational physicians, translated into ICD10 codes. Included were long-term SA due to mental and behavioral disorders (ICD-10 chapter V) and musculoskeletal and connective tissue diseases (ICD-10 chapter XIII).

\section{Predictor variables}

Age (in years), gender (men; women), education (low $=$ primary school and junior vocational education; medium $=$ secondary general and senior vocational education; high $=$ higher professional and academic education), and marital status (single; married; other, e.g., living with family) were obtained from the survey questionnaire.

Long-term SA ( $\geq 42$ consecutive days) in the year prior to the occupational health survey was retrieved from the occupational health register and used for the predictor variable 'prior long-term $\mathrm{SA}^{\prime}$ ' $(\mathrm{no}=0$, yes $=1)$.

Psychosocial work characteristics were measured with brief scales of the Questionnaire on the Experience and Evaluation of Work (QEEW) addressing work pace (5 items, Cronbach's $\alpha=0.87$ ), cognitive demands (5 items, $\alpha=0.83$ ), emotional demands ( 3 items, $\alpha=0.80$ ), work-home interference ( 7 items, $\alpha=0.88$ ), role clarity ( 5 items, $\alpha=0.85$ ), task variety (6 items, $\alpha=0.87$ ), learning opportunities ( 4 items, $\alpha=0.88$ ), supervisor support ( 3 items, $\alpha=0.90$ ), and co-worker support ( 3 items, $\alpha=0.89$ ) (Van Veldhoven and Meijman 1994). In line with the JD-R model, work pace, cognitive demands, emotional demands and work-home interference were considered job demands. The job resources were role clarity, task variety, learning opportunities, and support from supervisors and co-workers.

The occupational health survey questionnaire measured burnout with the 15-item Dutch version of the Maslach Burnout Inventory-general survey (MBI-GS) covering emotional exhaustion (feelings of being emotionally overextended and exhausted by one's work), cynicism (a feeling of distance and impersonal response towards recipients of one's care or service) and personal accomplishment (feelings of competence and successful achievement in one's work) (Bakker et al. 2002). All items had 7-point frequency response scales ranging from 'never' $(=0)$ to 'always' $(=6)$. Item scores were summed to a total MBI-GS score $(\alpha=0.89)$ and divided by the number of items so that burnout scores ranged between 0 and 6 . Higher scores represent higher levels of burnout.

Work engagement was measured with the 9-item Utrecht Work Engagement Scale (UWES) covering vigor (feeling strong and vigorous), dedication (enthusiasm about one's job and feeling proud and inspired) and absorption (feeling flow when working) with 7-point frequency scales ranging from 'never' $(=0)$ to 'always' $(=6)$ (Schaufeli et al. 2006). Item scores were summed to a total UWES score $(\alpha=0.94)$ and divided by the number of items so that work engagement scores ranged between 0 and 6 . Higher scores represent higher levels of work engagement. In the literature, burnout and work engagement are described as closely related concepts (Demerouti et al. 2010; Mäkikangas et al. 2012). In 
our study, burnout and work engagement were correlated (Pearson correlation $r=-0.488$ ), but not collinear.

\section{Statistical analyses}

Statistical analyses were done with $\mathrm{R}$ for Windows (version 3.2.4) using the Regression Modelling Strategies (rms) package (version 5.1-1) (https://cran.r-project.org/web/ packages/rms/rms.pdf). Age, job characteristics, burnout, and engagement were distributed normally. Student's $t$ tests for independent samples were used to determine differences in continuous baseline characteristics and Chi-square tests were used for the categorical variables. Gender, education, marital status, and prior long-term SA were included as categorical variables; age, work pace, workload, emotional demands, work-home interference, role clarity, task variety, learning opportunities, supervisor support co-worker support, burnout, and work engagement were included as continuous variables into logistic regression models with longterm SA $($ no $=0$, yes $=1)$ in the year following the survey as outcome variable. Model 1 included age, gender, education, marital status, prior long-term SA, job demands, and job resources. Model 2 included age, gender, education, marital status, prior long-term SA, burnout and work engagement. Logistic regression analysis estimated odds ratios (OR) and related $95 \%$ confidence intervals $(\mathrm{CI})$. The Wald-statistic is calculated using the formula $(B / \mathrm{SE})^{2}$ where $B$ is the regression coefficient and SE the standard error; higher Wald-statistics indicate stronger predictors. The prediction model was reduced by backward stepwise techniques, using Akaike's information criterion (AIC) as a stopping rule.

The overall predictive performance of the final model was assessed by the Nagelkerke's pseudo $R^{2}$. Calibration refers to the agreement between predicted and observed risks and was investigated with the Hosmer-Lemeshow (H-L) goodnessof-fit test. $\mathrm{H}-\mathrm{L}$ test $p \geq 0.05$ indicates that the predicted risks do not deviate significantly from the observed risks, meaning that risk predictions are adequate. Discrimination refers to the ability of a prediction model to distinguish between frequent absentees with and without long-term SA during follow-up. Discrimination was investigated by receiver operating characteristic (ROC) analysis. The area under the ROC-curve (AUC) was used as measure of discrimination; AUC $\geq 0.75$ represents discrimination useful for practice (Steyerberg 2009). All final models were stratified by gender. We also tested performance of the final models differentiating between sickness absence cause.

A prediction model will perform better in the subjects used to develop the model than in new subjects, a phenomenon known as overfitting. Overfitted prediction models are too optimistic in predicting outcomes for new subjects. Therefore, we internally validated the prediction models in
250 bootstrap samples to correct for over-optimistic predictions in new subjects.

\section{Results}

The frequent absentees with complete data $(n=3563)$ were significantly older, higher educated, had significantly lower work engagement and significantly more frequently prior long-term than those excluded because of missing responses on the occupational health survey questionnaires $(n=641)$. Gender, marital status, prior SA frequency, job demands, job resources and burnout did not differ significantly between included and excluded participants (Table 1).

During 1-year follow-up, 685 (19\%) frequent absentees had long-term SA, predominantly due to musculoskeletal $(n=319 ; 47 \%)$ and mental $(n=256 ; 37 \%)$ disorders; 15 participants had both mental long-term SA and musculoskeletal long-term SA in the follow-up year. Other causes of longterm SA were cardiovascular (6\%), gastro-intestinal (4\%), neurological (4\%), and various other disorders $(2 \%)$.

\section{Performance of prediction model 1, with job demands and job resources}

The full model 1 included 14 predictor variables. Based on the Wald-statistic, lower education, older age and female gender were the strongest predictors of long-term SA among frequent absentees. After backward stepwise reduction, 8 variables remained in the final model 1: age, gender, education, marital status, prior long-term SA, work pace, role clarity, and learning opportunities (Table 2).

The Nagelkerke's pseudo $R^{2}$ was 0.048 , reflecting poor overall performance of the final logistic regression model. The $\mathrm{H}-\mathrm{L}$ test $p=0.013$ indicated that the risks predicted by the model deviated significantly from the observed risks of long-term SA, although inspection of the calibration plot showed no substantial deviations between predicted and observed long-term SA risks (Fig. 2). The full 14-predictor model had an AUC 0.625 (95\% CI 0.599-0.654) and the final 8-predictor model 1 had AUC 0.623 (95\% CI 0.601-0.646). Bootstrapping showed $4.8 \%$ over-optimism; the over-optimism adjusted AUC of the final model 1 was 0.615 . Discrimination by the final model 1 did not differ between men (AUC 0.644; 95\% CI 0.617-0.671) and women (AUC 0.622; 95\% CI 0.583-0.660). Calibration was adequate for both men $(\mathrm{H}-\mathrm{L}$ test $p=0.104)$ and women $(\mathrm{H}-\mathrm{L}$ test $p=0.366$ ). The Nagelkerke's pseudo $R^{2}$ was 0.064 for men and 0.043 for women. Age, gender, education, marital status, and prior long-term SA were strong predictors in the final model. When stratified, age, education, marital status and prior long-term SA were also strong predictors 
Table 1 Characteristics of the study population $(N=4204)$

\begin{tabular}{|c|c|c|c|c|c|c|c|c|c|}
\hline & \multicolumn{4}{|c|}{$\begin{array}{l}\text { Included participants } \\
(n=3563)\end{array}$} & \multicolumn{4}{|c|}{$\begin{array}{l}\text { Excluded participants }^{\mathrm{a}} \\
(n=641)\end{array}$} & \multirow[t]{2}{*}{ Analysis } \\
\hline & Mean & $\mathrm{SD}^{\mathrm{c}}$ & $n$ & $\%$ & Mean & SD & $n$ & $\%$ & \\
\hline Age (years) & 44.2 & 10.9 & & & 43.0 & 10.7 & & & $p=0.014$ \\
\hline \multicolumn{10}{|l|}{ Gender } \\
\hline Men & & & 2399 & 67 & & & 398 & 62 & \multirow[t]{3}{*}{$p=0.063$} \\
\hline Women & & & 1164 & 33 & & & 223 & 35 & \\
\hline Missing & & & 0 & & & & 20 & 3 & \\
\hline \multicolumn{10}{|l|}{ Education } \\
\hline Low & & & 800 & 22 & & & 174 & 27 & \multirow[t]{4}{*}{$p=0.002$} \\
\hline Medium & & & 1618 & 45 & & & 248 & 39 & \\
\hline High & & & 1145 & 32 & & & 176 & 27 & \\
\hline Missing & & & 0 & & & & 43 & 7 & \\
\hline \multicolumn{10}{|l|}{ Marital status } \\
\hline Single & & & 898 & 25 & & & 156 & 24 & \multirow[t]{4}{*}{$p=0.609$} \\
\hline Married & & & 2524 & 71 & & & 403 & 63 & \\
\hline Other & & & 141 & 4 & & & 26 & 4 & \\
\hline Missing & & & 0 & & & & 56 & 9 & \\
\hline \multicolumn{10}{|l|}{ Job demands (range 1-5) } \\
\hline Work pace & 2.7 & 0.9 & & & 2.7 & 0.9 & & & $p=0.892$ \\
\hline Cognitive demands & 3.5 & 0.8 & & & 3.5 & 0.8 & & & $p=0.266$ \\
\hline Emotional demands & 1.7 & 0.7 & & & 1.7 & 0.7 & & & $p=0.842$ \\
\hline Work-home interference & 1.6 & 0.6 & & & 1.6 & 0.6 & & & $p=0.932$ \\
\hline \multicolumn{10}{|l|}{ Job resources (range $1-5$ ) } \\
\hline Role clarity & 3.9 & 0.8 & & & 3.9 & 0.8 & & & $p=0.953$ \\
\hline Task variety & 3.4 & 0.9 & & & 3.4 & 0.8 & & & $p=0.675$ \\
\hline Learning opportunities & 2.8 & 1.0 & & & 2.8 & 1.0 & & & $p=0.897$ \\
\hline Supervisor support & 3.5 & 1.1 & & & 3.5 & 1.1 & & & $p=0.929$ \\
\hline Co-worker support & 3.8 & 0.9 & & & 3.8 & 0.9 & & & $p=0.513$ \\
\hline Burnout (range 0-6) & 2.3 & 0.5 & & & 2.3 & 0.7 & & & $p=0.119$ \\
\hline Work engagement (range 0-6) & 3.5 & 1.1 & & & 3.7 & 1.1 & & & $p=0.001$ \\
\hline \multicolumn{10}{|c|}{ Sickness absence spells in the year prior to the survey } \\
\hline 3 & & & 2031 & 57 & & & 337 & 53 & \multirow[t]{5}{*}{$p=0.073$} \\
\hline 4 & & & 879 & 25 & & & 165 & 26 & \\
\hline 5 & & & 353 & 10 & & & 66 & 10 & \\
\hline 6 & & & 168 & 5 & & & 45 & 7 & \\
\hline$>6$ & & & 132 & 4 & & & 28 & 4 & \\
\hline \multicolumn{10}{|c|}{ Long-term sickness absence in the year prior to the survey } \\
\hline No & & & 2648 & 74 & & & 543 & 85 & \multirow[t]{2}{*}{$p=0.000$} \\
\hline Yes & & & 915 & 26 & & & 98 & 15 & \\
\hline \multicolumn{10}{|c|}{ Long-term sickness absence in the year following the survey } \\
\hline No & & & 2878 & 81 & & & 520 & 81 & \multirow[t]{2}{*}{$p=0.870$} \\
\hline Yes & & & 685 & 19 & & & 121 & 19 & \\
\hline
\end{tabular}

The table shows the characteristics of the participants in occupational health questionnaires who had three or more SA spells in the year prior to the survey. The table compares the baseline characteristics of included and excluded participants

${ }^{a}$ Exclusion because of missing responses on the baseline predictor variables

${ }^{\mathrm{b}}$ Analysis of difference between included and excluded participants; Student's $t$ test for independent samples for continuous variables and Chi-square test for categorical variables

${ }^{\mathrm{c}} S D$ standard deviation 
Table 2 Prediction model on all-cause long-term sickness absence with job demands and job resources (model 1)

\begin{tabular}{|c|c|c|c|c|c|c|c|c|}
\hline & \multirow{2}{*}{\multicolumn{2}{|c|}{$\frac{\text { Full model }}{\text { All }(n=3563)}$}} & \multicolumn{6}{|c|}{ Final model } \\
\hline & & & \multicolumn{2}{|c|}{ All $(n=3563)$} & \multicolumn{2}{|c|}{ Men $(n=2399)$} & \multicolumn{2}{|c|}{ Women $(n=1164)$} \\
\hline & Wald & OR $(95 \% \mathrm{CI})$ & Wald & OR $(95 \% \mathrm{CI})$ & Wald & OR $(95 \%$ CI $)$ & Wald & OR $(95 \% \mathrm{CI})$ \\
\hline Age & 17.36 & $1.02(1.01-1.03)$ & 17.75 & $1.02(1.01-1.03)$ & 24.66 & $1.03(1.02-1.04)$ & 0.05 & $1.00(0.99-1.02)$ \\
\hline Gender & 17.14 & & 17.49 & & - & & - & \\
\hline Men & & 1 & & 1 & & - & & - \\
\hline Women & & $1.49(1.23-1.80)$ & & $1.49(1.24-1.79)$ & & - & & - \\
\hline Education & 25.08 & & 26.05 & & 16.14 & & 10.99 & \\
\hline Low & & 1 & & 1 & & 1 & & 1 \\
\hline Medium & & $0.75(0.61-0.92)$ & & $0.75(0.61-0.92)$ & & $0.77(0.60-0.99)$ & & $0.70(0.48-1.03)$ \\
\hline High & & $0.53(0.41-0.68)$ & & $0.53(0.41-0.67)$ & & $0.52(0.38-0.72)$ & & $0.50(0.33-0.76)$ \\
\hline Marital status & 5.90 & & 6.09 & & 6.81 & & 1.18 & \\
\hline Single & & 1 & & 1 & & 1 & & 1 \\
\hline Married & & $0.85(0.70-1.03)$ & & $0.84(0.69-1.02)$ & & $0.76(0.59-0.98)$ & & $0.88(0.65-1.19)$ \\
\hline Other & & $0.54(0.30-0.97)$ & & $0.54(0.30-0.97)$ & & $0.47(0.22-1.02)$ & & $0.66(0.26-1.69)$ \\
\hline Prior long-term SA & 8.34 & & 8.50 & & 10.97 & & 0.10 & \\
\hline No & & 1 & & 1 & & 1 & & 1 \\
\hline Yes & & $1.32(1.09-1.59)$ & & $1.32(1.10-1.59)$ & & $1.48(1.17-1.87)$ & & $1.05(0.76-1.45)$ \\
\hline Work pace & 2.51 & $1.10(0.98-1.24)$ & 2.41 & $1.09(0.98-1.21)$ & 1.87 & $1.09(0.96-1.24)$ & 3.03 & $1.16(0.98-1.36)$ \\
\hline Cognitive demands & 0.27 & $0.97(0.85-1.10)$ & & & & & & \\
\hline Emotional demands & 0.37 & $1.04(0.91-1.20)$ & & & & & & \\
\hline Work-home interference & 0.34 & $1.04(0.90-1.21)$ & & & & & & \\
\hline Role clarity & 2.54 & $0.90(0.78-1.03)$ & 2.67 & $0.90(0.80-1.02)$ & 0.25 & $1.04(0.89-1.22)$ & 12.23 & $0.70(0.58-0.86)$ \\
\hline Task variety & 0.08 & $0.98(0.86-1.12)$ & & & & & & \\
\hline Learning opportunities & 2.42 & $0.91(0.81-1.03)$ & 3.96 & $0.91(0.83-1.00)$ & 3.73 & $0.89(0.79-1.00)$ & 1.57 & $0.91(0.77-1.06)$ \\
\hline Supervisor support & 0.20 & $0.98(0.88-1.08)$ & & & & & & \\
\hline Support co-workers & 1.95 & $1.08(0.97-1.21)$ & & & & & & \\
\hline
\end{tabular}

The table shows Wald characteristics as indicator of predictor strength and the odds ratio $(O R)$ and 95\% confidence interval $(C I)$ of associations between the health survey variables and all-cause long-term sickness absence $(S A)$ for the full 14-predictor model and the final 8-predictor model obtained by backward stepwise statistical reduction. The final model was stratified by gender

for men. For women, education and role clarity were strong predictors.

\section{Performance of prediction model 2, with burnout and work engagement}

The full model 2 included 7 variables: age, gender, education, marital status, prior long-term SA, burnout, and work engagement. Again, lower education, older age, and female gender were the strongest predictors of long-term SA. After backward stepwise reduction, all 7 predictor variables remained in the final model 2 (Table 3 ).

The overall predictive performance of the model was poor, with Nagelkerke's pseudo $R^{2}=0.044$. An H-L test $p=0.009$ indicated miscalibration, although the calibration plot showed no substantial deviations between predicted and observed long-term SA risks (Fig. 2). The discriminative ability of the model was significant with AUC 0.624 (95\% CI 0.596-0.651); after correction for
5.2\% over-optimism, the AUC was 0.616. The final predictor model had AUC 0.646 (95\% CI 0.619-0.673) for men, and AUC 0.583 (95\% CI 0.544-0.622) for women. Calibration was adequate for the model with men and women with $\mathrm{H}-\mathrm{L}$ test $p=0.436$ and $p=0.632$, respectively. The Nagelkerke's pseudo $R^{2}$ was 0.063 for men and 0.021 for women. For male frequent absentees, age, gender, education, marital status, prior long-term SA, and burnout were strong predictors of long-term SA, whereas for women education was the strongest predictor.

Table 4 shows the results of the final prediction models differentiated by sickness absence causes. When modelling only on participants with long-term SA due to mental disorders, discriminative ability was significant with AUC 0.635 (95\% CI 0.599-0.670) for model 1 and AUC 0.610 (95\% CI 0.574-0.646) for model 2. Discrimination was better, but still not useful for practice when modelling only on participants with long-term SA due to musculoskeletal disorders, with AUC 0.688 (95\% CI 0.660-0.716) for 
Fig. 2 Calibration graph. The figure plots mean long-term SA risks predicted by the final 8-predictor model with job demand job resources model (black dots) and the final 7-predictor model with burnout and work engagement (grey dots) against observed frequencies per decile of predicted risk; the diagonal indicates perfect calibration

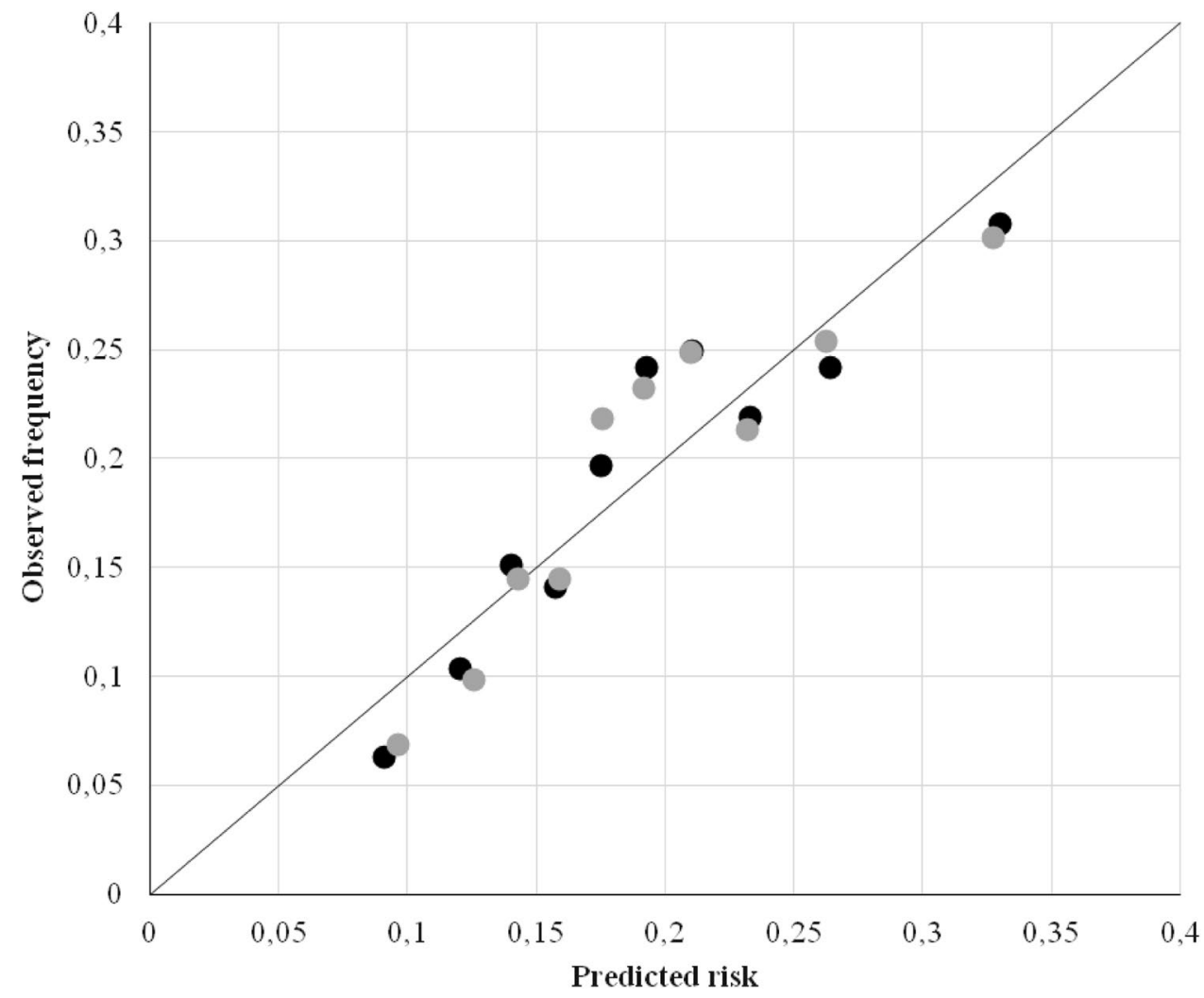

\begin{tabular}{|c|c|c|c|c|c|c|}
\hline & \multicolumn{2}{|c|}{ All $(n=3563)$} & \multicolumn{2}{|c|}{$\operatorname{Men}(n=2399)$} & \multicolumn{2}{|c|}{ Women $(n=1164)$} \\
\hline & Wald & OR $(95 \% \mathrm{CI})$ & Wald & OR $(95 \% \mathrm{CI})$ & Wald & OR $(95 \% \mathrm{CI})$ \\
\hline Age & 16.98 & $1.02(1.01-1.03)$ & 24.73 & $1.03(1.02-1.04)$ & 0.06 & $1.00(0.98-1.01)$ \\
\hline Gender & 16.96 & & - & & - & \\
\hline Men & & 1 & & - & & - \\
\hline Women & & $1.51(1.26-1.81)$ & & - & & - \\
\hline Education & 27.17 & & 19.14 & & 9.53 & \\
\hline Low & & 1 & & 1 & & 1 \\
\hline Medium & & $0.72(0.59-0.89)$ & & $0.75(0.58-0.96)$ & & $0.71(0.49-1.04)$ \\
\hline High & & $0.53(0.32-0.67)$ & & $0.50(0.37-0.68)$ & & $0.54(0.36-0.80)$ \\
\hline Marital status & 5.50 & & 7.14 & & 1.22 & \\
\hline Single & & 1 & & 1 & & 1 \\
\hline Married & & $0.86(0.71-1.04)$ & & $0.75(0.58-0.97)$ & & $0.91(0.67-1.22)$ \\
\hline Other & & $0.50(0.28-0.91)$ & & $0.46(0.21-0.99)$ & & $0.61(0.24-1.56)$ \\
\hline Prior long-term SA & 8.96 & & 10.69 & & 0.00 & \\
\hline No & & 1 & & 1 & & 1 \\
\hline Yes & & $1.32(1.10-1.57)$ & & $1.47(1.17-1.86)$ & & $1.00(0.73-1.38)$ \\
\hline Burnout & 6.55 & $1.22(1.05-1.42)$ & 5.87 & $1.26(1.05-1.53)$ & 1.01 & $1.15(0.87-1.52)$ \\
\hline Work engagement & 1.78 & $0.95(0.88-1.02)$ & 0.30 & $0.97(0.89-1.07)$ & 2.69 & $0.89(0.78-1.02)$ \\
\hline
\end{tabular}

The table shows Wald characteristics as indicator of predictor strength and the odds ratio $(O R)$ and $95 \%$ confidence interval $(C I)$ of associations between the health survey variables and all-cause long-term sickness absence. The model including men and women combined (all) concerns the full and final 7-predictor model. The model was stratified by gender 
Table 4 Results per model for long-term SA due to different causes

\begin{tabular}{llll}
\hline & $\begin{array}{l}\text { Nagelkerke's pseudo } \\
R^{2}\end{array}$ & $\mathrm{H}-\mathrm{L}$ test & AUC (95\% CI) \\
\hline Model 1 & & & \\
$\quad$ All cause long-term SA & 0.048 & 0.013 & $0.623(0.601-0.646)$ \\
Mental long-term SA & 0.040 & 0.712 & $0.635(0.599-0.670)$ \\
Musculoskeletal long-term SA & 0.079 & 0.866 & $0.688(0.660-0.716)$ \\
Model 2 & & & \\
All cause long-term SA & 0.044 & 0.009 & $0.624(0.596-0.651)$ \\
Mental long-term SA & 0.030 & 0.815 & $0.610(0.574-0.646)$ \\
Musculoskeletal long-term SA & 0.071 & 0.730 & $0.679(0.650-0.707)$ \\
\hline
\end{tabular}

The table presents prediction model performance measures differentiated by sickness absence cause; $\mathrm{H}-\mathrm{L}$ test $p \geq 0.05$ indicates adequate model calibration; the area under the receiver operating characteristic curve $(A U C)$ reflects discrimination by the model between frequent absentees with and without long-term sickness absence during follow-up model 1 and AUC 0.679 (95\% CI 0.650-0.707) for model 2 .

\section{Discussion}

We developed prediction models for the risk of long-term SA among frequent absentees using backward stepwise regression analysis. Final model 1 included age, gender, education, marital status, prior long-term SA, work pace, role clarity and learning opportunities. Discrimination by this model between frequent absentees with and without longterm SA during follow-up was significant, but not useful for practice. Model 2 included age, gender, education, marital status, prior long-term SA, burnout and work engagement. Discrimination between frequent absentees with and without long-term SA during follow-up was comparable to model 1. We hypothesized that it would be unfeasible to measure all potentially important job demands and job resources and therefore expected a better performance of model 2 as compared to model 1. Model 1 and model 2 showed comparable performance, even when stratifying the performance analysis by gender or when differentiating between long-term SA causes. Model 1 correctly identified frequent absentees with long-term SA during 1-year follow-up in $61.5 \%$ of the cases and model 2 in $61.6 \%$ of the cases. Although better than chance, discrimination of this magnitude is below the level recommended for practical use.

The poor discriminative ability is in line with previous research on prediction models for long-term SA. A prediction model including age, gender, education, self-rated health, mental health, prior long-term SA, work ability, emotional job demands, and recognition by the management correctly identified Danish employees at risk of long-term SA in $68 \%$ of the cases (Roelen et al. 2018). In a study on employees of an airline company, Boot et al. (2017) found that higher age, recent pregnancy, having a parking permit, having 'aggravated working conditions' (i.e., physical workload as a result of posture, lifting and abnormal working conditions) and prior SA correctly identified employees at risk of long-term SA in $73 \%$ of the cases. The better discriminative ability may be due to the fact that predictions were restricted to employees of one company, which enabled the investigators to include specific predictors, such as 'having a parking permit'.

A recent study on prediction models including job demands and job resources showed poor discrimination between employees with and without long-term SA (Roelen et al. 2017). The prediction model, including psychological job demands, role conflict, harassment, role clarity, social support and fair leadership at the workplace, correctly identified nurses with long-term SA during 2-year follow-up in $56 \%$ of the cases. The explanation for the poor discriminative ability of this prediction model may be that the association of job demands and job resources with health outcomes differs across workplace settings; demands or resources that have a strong association with long-term SA in one workplace might be weakly or not associated with long-term SA in another workplace. Furthermore, there may be unknown job demands and job resources that are important predictors of long-term SA. Knowing we could not include all possible job demands and resources for all types of jobs and industries, we expected better predictions by the model including burnout and work engagement, because sustained high levels of both measured and unmeasured job demands will lead to burnout. Although the present study showed that higher burnout scores were associated with an increased long-term SA risk, discrimination between frequent absentees with and without long-term SA by the prediction model including burnout and work engagement was not better than discrimination by the model including job demands and job resources. Although both prediction models included psychosocial work factors, discrimination was not better for long-term SA due to mental disorders than for long-term SA 
due to musculoskeletal disorders. When stratifying the final models by gender, discrimination was comparable. These finding indicate that neither the model with job demands and job resources, nor the model with burnout and work engagement discriminates sufficiently between frequent absentees with and without long-term SA during 1-year follow-up. It is unlikely that longer follow-up periods improve the discrimination of baseline predictor models (Airaksinen et al. 2018). Although longer follow-up periods result in more events and higher statistical power, baseline predictor models predict outcomes most accurately on the short term (Melloh et al. 2012), particularly if predictor values change over time. Longitudinal prediction models with repeated measurements of predictor values over time may better discriminate between frequent absentees with and without long-term SA.

\section{Strengths and weaknesses}

To our knowledge, this is the first cohort study investigating predictions of long-term SA among frequent absentees. Job demands, job resources, burnout and work engagement were all measured at baseline with reliable and valid scales and the analysis was based on the JD-R model as a theoretical framework (Van Veldhoven and Meijman 1994; Bakker et al. 2002; Schaufeli et al. 2006). Including burnout and work engagement could have improved the model (Borritz et al. 2010; Rongen et al. 2015), but due to the health impairment process described by the JD-R model, we decided to analyse job demands and job resources separately from burnout and work engagement. The data of the frequent absentees were obtained from a large population $(N=53,833)$ of employees who participated in occupational health surveys between 2010 and 2013. With 685 long-term SA episodes at follow-up and 14 variables in the full model we had almost 50 events per variable, which was sufficient for a robust backward regression analysis. Participants in health surveys may be healthier than non-participants (Froom et al. 1999). Healthy volunteer bias may have under-estimated associations between predictor variables and long-term SA, if healthy frequent absentees participated in health surveys more often than those with chronic health conditions. Although participants differed on some characteristics from those excluded at baseline, in most cases, the difference was small in absolute numbers except for prior long-term SA: $26 \%$ of the participants reported prior long-term SA as compared with $15 \%$ of those excluded at baseline. This may have resulted in overestimation of associations between prior long-term SA and long-term SA at follow-up. Selective participation may hamper the generalizability of the results.

The low Nagelkerke's pseudo- $R^{2}$ values indicate that important predictors of long-term SA among frequent absentees may be lacking from the prediction models. An earlier study (Slany et al. 2014) found indications that job demands and job resources predictive of long-term SA may differ between men and women. When stratifying our final models by gender, we also found that the predictor strength of several factors in women was different from men. Age, marital status and prior long-term SA were stronger predictors of long-term SA in male frequent absentees than in female frequent absentees. Role clarity was a strong predictor for women. However, the predictive performance of the models did not differ between men and women.

\section{Practical implications}

The present study showed that education, age and gender were the strongest predictors of long-term SA among frequent absentees. We recommend health providers and managers to explore the causes of frequent SA in low educated, older and female frequent absentees as they are particularly at risk of long-term SA. With the current knowledge, this may be the best strategy for preventing long-term SA among frequent absentees. A prediction model for long-term SA would enable healthcare providers to better identify frequent absentees at increased risk of long-term SA and invite them for preventive consultations or refer them to interventions to reduce the risk of long-term SA. Prediction models including job demands and job resources or their effects in terms of burnout and work engagement proved to be better than chance, but have to be further developed for use in healthcare practice.

\section{Further research}

The poor performance of the prediction models in the present study indicates that important predictors of longterm SA may be lacking from the models. More research is required to search for additional predictors of long-term SA among frequent absentees. Previous studies have included health-related predictors (Roelen et al. 2018; Boot et al. 2017; Laaksonen et al. 2011). Roskes et al. (2005) have reported that employees with chronic conditions have more frequent SA. Health-related variables may improve the predictions of long-term SA among frequent absentees. Furthermore, several studies have shown that work ability is a predictor of future long-term SA (Roelen et al. 2018; Reeuwijk et al. 2015; Schouten et al. 2015, 2016). We did not include work ability in the present study, because it was not measured in all occupational health surveys. Previous studies have shown that influence at work and quality of leadership predict long-term SA. Future studies could investigate if the prediction model for long-term SA among frequent absentees improves by adding work ability, health related variables such as self-rated health, influence at work or quality of leadership as a predictor variable. Furthermore, future studies should consider developing prediction models for 
men and women, as our present results show that predictors of long-term sickness absence differ between male and female frequent absentees. Age and prior long-term SA may not be included as predictor variables in the final prognostic model for long-term sickness absence in female frequent absentees.

\section{Conclusion}

A prediction model including job demands and job resources and a prediction model including burnout and work engagement better than chance discriminated between frequent absentees with and without long-term SA during 1-year follow-up, but have to be further developed before using them to identify frequent absentees at risk of long-term SA and refer them to interventions aimed at preventing longterm SA.

\section{Compliance with ethical standards}

Ethical standards All procedures performed in studies involving human participants were in accordance with the ethical standards of the institutional and/or national research committee and with the 1964 Helsinki declaration and its later amendments or comparable ethical standards. The Medical Ethics Committee of the University Medical Center Groningen granted ethical clearance for this study (M12.116654).

Conflict of interest The authors declare that they have no conflict of interest.

Open Access This article is distributed under the terms of the Creative Commons Attribution 4.0 International License (http://creativeco mmons.org/licenses/by/4.0/), which permits unrestricted use, distribution, and reproduction in any medium, provided you give appropriate credit to the original author(s) and the source, provide a link to the Creative Commons license, and indicate if changes were made.

\section{References}

Airaksinen J, Jokela M, Virtanen M, Oksanen T, Koskenvuo M, Pentti J, Vahtera J, Kivimäki M (2018) Prediction of long-term absence due to sickness in employees. Scand J Work Environ Health 44:274-282

Bakker AB, Demerouti E (2007) The job demands-resources model: state of the art. J Manag Psychol 22:309-328

Bakker AB, Demerouti E, Schaufeli WB (2002) Validation of the Maslach Burnout Inventory-general survey: an internet study. Anxiety Stress Coping 15:245-260

Bakker AB, Demerouti E, Boer de E, Schaufeli WB (2003) Job demands and job resources as predictors of absence duration and frequency. J Vocat Behav 62:341-356

Boot CRL, van Drongelen A, Wolbers I, Hlobil H, van der Beek AJ, Smid T (2017) Prediction of long-term and frequent sickness absence using company data. Occup Med 67:176-181

Borritz M, Christensen KB, Bültmann U, Rugulies R, Lund T, Andersen I, Villadsen E, Diderichsen F, Kristensen TS (2010)
Impact of burnout and psychosocial work characteristics on future LT sickness absence. Prospective results of the Danish PUMA study among human service workers. J Occup Environ Med 52:964-970

Clausen T, Nielsen K, Carneiro IG, Borg V (2012) Job demands, job resources and long-term sickness absence in the Danish eldercare services: a prospective analysis of register-based outcomes. J Adv Nurs 68:127-136

Clausen T, Burr H, Borg V (2014) Do psychosocial job demands and job resources predict long-term sickness absence? An analysis of register-based outcomes using pooled data on 39,408 individuals in four occupational groups. Int Arch Occup Environ Health 87:909-917

Demerouti E, Mostert K, Bakker AB (2010) Burnout and work engagement: a thorough investigation of the independency of both constructs. J Occup Health Psychol 15:209-222

Eriksson UB, Starrin B, Janson S (2008) Long-term sickness absence due to burnout: absentees' experiences. Qual Health Res 18:620-632

Eriksson UB, Engström LG, Starrin B, Janson S (2011) Insecure social relations at work and LT sickness absence due to burnout and other mental diagnoses. Work 38:319-327

Froom P, Melamed S, Kristal-Boneh E, Benbassat J, Ribak J (1999) Healthy volunteer effect in industrial workers. J Clin Epidemiol 52:731-735

Koopmans PC, Roelen CA, Groothoff JW (2008a) Risk of future sickness absence in frequent and LT absentees. Occup Med 58:268-274

Koopmans PC, Roelen CAM, Groothoff JW (2008b) Frequent and long-term absence as a risk factor for work disability and job termination among employees in the private sector. Occup Environ Med 65:494-499

Laaksonen M, Kääriä SM, Leino-Arjas P, Lahelma E (2011) Different domains of health functioning as predictors of sickness absence-a prospective cohort study. Scand J Work Environ Health 37:213-221

Mäkikangas A, Feldt T, Kinnunen U, Tolvanen A (2012) Do low burnout and high work engagement always go hand in hand? Investigation of the energy and identification dimensions in longitudinal data. Anxiety Stress Coping 25:93-116

Melloh M, Elfering A, Salathe CR, Kaser A, Barz T, Röder C, Theis JC (2012) Predictors of sickness absence in patients with a new episode of low back pain in primary care. Ind Health 50:288-298

Reeuwijk KG, Robroek SJ, Niessen MA, Kraaijenhagen RA, Vergouwe Y, Burdorf A (2015) The prognostic value of the work ability index for sickness absence among office workers. PLoS One 10(5):e0126969

Roelen CAM, van Hoffen MFA, Groothoff JW, de Bruin J, Schaufeli WB, van Rhenen W (2015) Can the Maslach Burnout Inventory and Utrecht Work Engagement Scale be used to screen for risk of long-term sickness absence? Int Arch Occup Environ Health $88: 467-475$

Roelen CAM, van Hoffen MFA, Waage S, Schaufeli WB, Twisk JWR, Bjorvatn B, Moen BE, Pallesen S (2017) Psychosocial work environment and mental-health related long-term sickness absence among nurses. Int Arch Occup Environ Health. https ://doi.org/10.1007/s00420-017-1268-1. (Epub 2017 Oct 14)

Roelen C, Thorsen S, Heymans M, Twisk J, Bültmann U, Bjørner J (2018) Development and validation of a prediction model for LT sickness absence based on occupational health survey variables. Disabil Rehabil 40:168-175

Rongen A, Robroek SJ, Schaufeli W, Burdorf A (2015) The contribution of work engagement to self-perceived health, workability, and sickness absence beyond health behaviors and work-related factors. J Occup Environ Med 56:892-897 
Roskes K, Donders NCGM, van der Gulden JWJ (2005) Healthrelated and work-related aspects associated with sick leave: a comparison of chronically ill and non-chronically ill workers. Int Arch Occup Environ Health 78:270-278

Schaufeli WB, Bakker AB (2004) Job demands, job resources, and their relationship with burnout and engagement: a multi-sample study. J Organ Behav 25:293-315

Schaufeli WB, Bakker AB, Salanova M (2006) The measurement of work engagement with a short questionnaire: a cross-national study. Educ Psychol Meas 66:701-716

Schaufeli WB, Bakker AB, van Rhenen W (2009) How changes in job demands and resources predict burnout, work engagement, and sickness absenteeism. J Org Behav 30:893-917

Schouten LS, Joling C, van der Gulden JWJ, Heymans MW, Bültmann U, Roelen CAM (2015) Screening manual and office workers for risk of long-term sickness absence: cut-off points for the Work Ability Index. Scand J Work Environ Health 41:36-42

Schouten LS, Bültmann U, Heymans MW, Joling CI, Twisk JW, Roelen CA (2016) Shortened version of the work ability index to identify workers at risk of long-term sickness absence. Eur J of Public Health 26:301-305

Slany C, Schütte S, Chastang JF, Parent-Thirion A, Vermeylen G, Niedhammer I (2014) Psychosocial work factors and long sickness absence in Europe. Int J Occup Environ Health 20:16-25

Stapelfeldt CM, Nielsen CV, Andersen NT, Krane L, Borg V, Fleten N, Jensen C (2014) Sick leave patterns as predictors of disability pension or LT sick leave: a 6.75-year follow-up study in municipal eldercare workers. BMJ Open 4:e003941

Steyerberg EW (2009) Clinical prediction models. Springer, New York

Strømholm T, Pape K, Ose SO, Krokstad S, Bjørngaard JH (2015) Psychosocial working conditions and sickness absence in a general population: a cohort study of 21,834 workers in Norway (The HUNT Study). J Occup Environ Med 57:386-392

Van Veldhoven M, Meijman T (1994) Het meten van psychosociale arbeidsbelasting met een vragenlijst. De Vragenlijst Beleving en Beoordeling van de Arbeid. NIA, Amsterdam 